\title{
Switching-Type Lambda Sensor Manufactured by Joining of Sintered Zirconia via Glass Solder Paste
}

\author{
Franz Schubert, Stefan Wollenhaupt, Jaroslaw Kita, Gunter Hagen, Ralf Moos \\ University of Bayreuth, Department of Functional Materials, 95440 Bayreuth, Germany \\ Franz.Schubert@Uni-Bayreuth.de
}

\begin{abstract}
:
This paper presents a planar switching-type lambda sensor fabricated by joining the heating and sensing components made of yttria-stabilized zirconia using a glass soldering paste. A way to produce a dense joining layer was found. The resulting sensor devices were compared with a sensor manufactured in a high temperature co-firing process and with a commercial sensor. The resulting sensor devices performed as expected and "switched" between lean and rich exhaust atmospheres.
\end{abstract}

\section{Introduction}

Typical oxygen sensors for harsh environments rely on yttria-stabilized zirconia (YSZ) as an ionic conductor and metallic electrodes being in contact with different gas atmospheres. Typically, this type of planar structure is selfheated, using a platinum heater [1-3]. Such sensors are usually manufactured in High Temperature Co-fired Ceramic (HTCC) technology, using a one-step firing process. Due to the high firing temperature (above $\left.1400{ }^{\circ} \mathrm{C}\right)$, the choice of electrode materials is practically limited to platinum. However, application of other materials would be interesting to alter the sensing properties to different gases and for cost reasons [4]. Since the variation of electrode materials in a sensor is not possible using HTCC process (most electrode materials would melt or decompose during firing), we developed a joining-technique for sintered YSZ structures.

\section{Experimental}

A switching-type lambda sensor was divided up in the two parts: a heating and a sensing component. Both were produced separately in HTCC technology and were joined using a glass solder. To manufacture the sensor components, the material system from ESL ElectroScience was used. It contains a $5 \mathrm{~mol} \%$ yttria-stabilized zirconia tape, an alumina paste to insulate the heater, a platinum paste for printing the heater and a porous platinum paste for printing the electrodes. Firing was accomplished at $1550{ }^{\circ} \mathrm{C}$ in a Nabertherm LHT 08/17. Cutting the green sheet to a pattern and insertion of vias was done by LPKF Microline 350L.
A sensor preparation scheme is shown in Fig. 1.

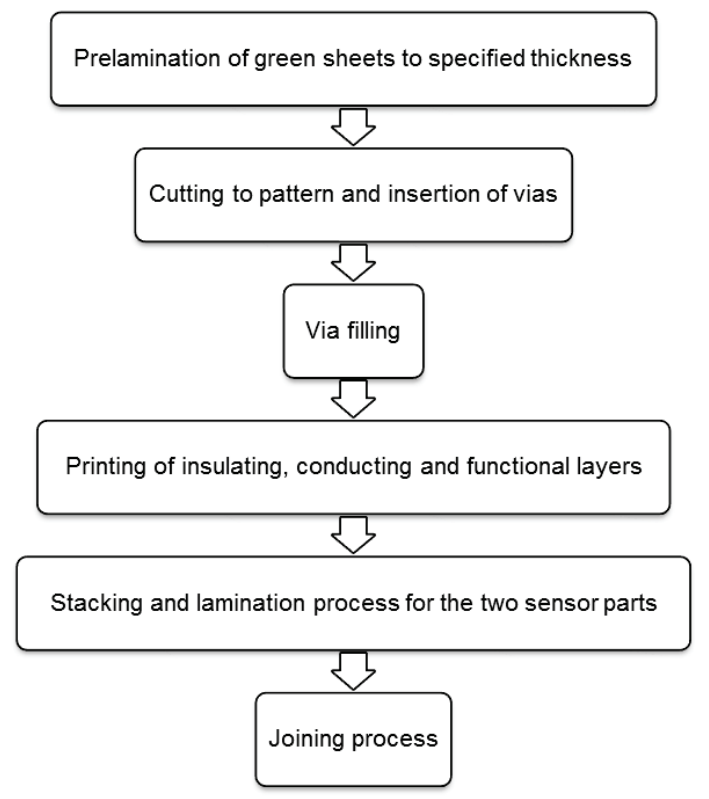

Fig. 1 Process to manufacture a joined switchingtype lambda sensor.

The solder was obtained by mixing commercial recrystallizing glass powder with thermal expansion coefficient (TCE) adjusted to YSZ ceramics with a binder made of ethyl cellulose and terpineol (ratio 1:11) using a three roll mill (EXAKT).

Two different types of glass powder (type $A$ und type B) were used for experiments. $T_{G}$ for type A was around $660{ }^{\circ} \mathrm{C}$ and for type $\mathrm{B}$ around $600{ }^{\circ} \mathrm{C}$. Both powders were sieved with a mesh width of $90 \mu \mathrm{m}$ to remove the coarsest particles. Additionally the glass powder was dried at $80^{\circ} \mathrm{C}$ for $8 \mathrm{~h}$. 
The prepared paste was screen printed for application and leveled for $15 \mathrm{~min}$. In first experiments, the paste was not dried before both sides were brought together so that the capillary force could reduce the space between the surfaces and compensate flatnessimperfections which can arise during manufacturing of the joining parts. After the parts were adhered, the sensor was dried at $100{ }^{\circ} \mathrm{C}$ for $2 \mathrm{~h}$. Then type $\mathrm{A}$ was sintered at $1000^{\circ} \mathrm{C}$ for $15 \mathrm{~min}$ and type $\mathrm{B}$ was sintered for $15 \mathrm{~min}$ at $850^{\circ} \mathrm{C}$. Both samples were weighted. The resulting sample was cut by a diamond saw and polished to investigate the joining layer by optical microscope.

In the following experiments the binder was dried before joining the parts. With respect to flatness and imperfections of the joining surfaces, the size of the solder was doubled by printing on both joining surfaces.

The resulting sensor was checked for tightness by dye penetrant inspection. The new sensor was compared with a sensor produced in a one-step sintering process. To the electrodes exposed to the test gas, a platinum catalyst on alumina carrier was applied by brush coating to enhance chemical equilibrium kinetics.

Both self-heated sensors were compared with a commercial type lambda probe with respect to their sensitivity to lambda changes (at around the stoichiometric point at $\lambda=1$ ). The resistances of the heaters of the laboratory-produced devices were calibrated in the furnace. The characteristic curve was then used to adjust the sensor to the desired temperature. A Keithley 2700 was used to log the Nernst potentials between the electrodes. The sensor produced in one-step sintering was measured at $650{ }^{\circ} \mathrm{C}$, whereas the other sensors were measured at $720^{\circ} \mathrm{C}$.

\section{Results and discussion}

Best results for screen printing were obtained for pastes with glass contents of $70 \mathrm{wt} \%$, which gave a suitable viscosity for printing. The appearance of powder and paste can be seen in Fig. 2.

a)

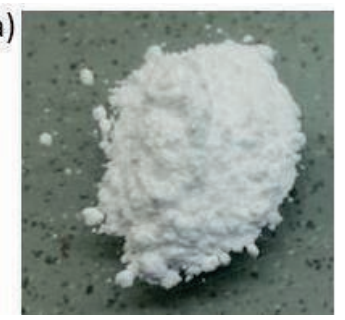

Fig. 2 The glass powder (a)) and paste (b)).
Fig. 3 shows the results for both glass types. The layers are mechanically stable even when they are cut. Type A shows many pores with sizes ranging from $50 \mu \mathrm{m}$ to $500 \mu \mathrm{m}$. Type $B$ also shows many pores, however the number of big pores is reduced. Whether this can be attributed to the glass itself, is not clear at the moment. Both glasses seem to be suitable to form a stable joint. Mainly due to its lower sintering temperature, type B was chosen for further investigation as this renders more different types of electrodes possible.

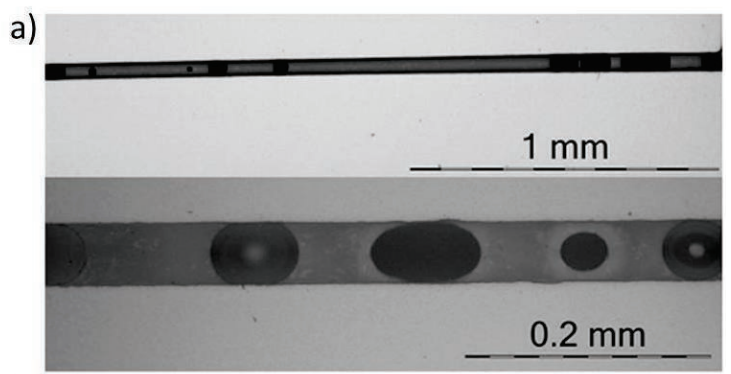

b)

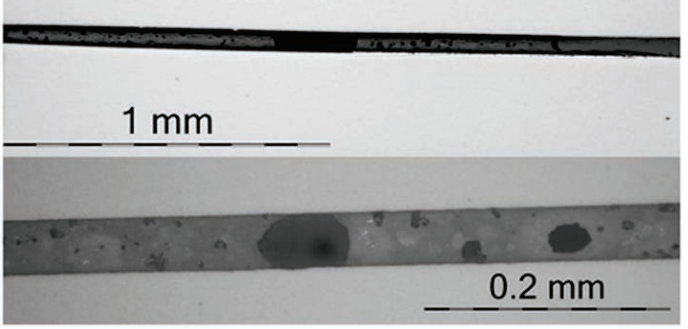

Fig. 3 Optical microscope picture of the joining layer of Type $A(a)$ ) and Type $B(b))$, when the joining occurs before drying.

Pores can be greatly reduced in number and size by drying of the paste before joining the layers (Fig. 4). Size is reduced for nearly all pores below $30 \mu \mathrm{m}$.
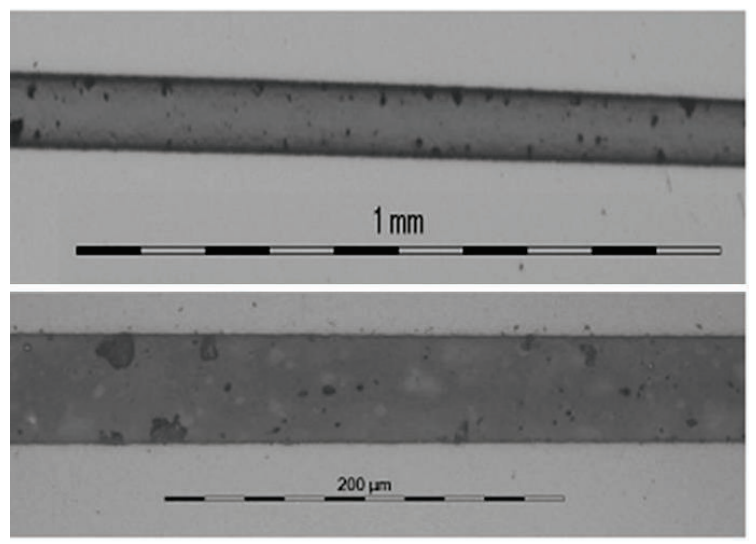

Fig. 4 Optical microscope pictures of the joining layer of Type $B$ when the layers are joined after drying of the paste.

Applying the solder by screen-printing allows for exact placement and leveling. Fig. 5 shows the two parts of the sensor with applied solder. On the left, the electrode and the reference 
chamber can be seen, as well as the reference channel and the conduction path for the electrodes. At the right end, there are contact pads for the sensor and the opening of the reference channel.

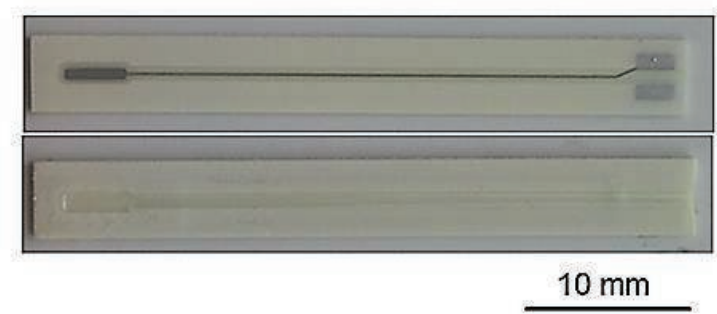

Fig. 5 Two parts of the sensor with glass paste applied to the joining surfaces.

Tightness of the sensor devices was proved by a dye impregnation test. After that, the sensor devices were mounted into a gas test bench. To achieve a gas-tight sealing, the sensor was cast integrally into a stainless steel tube by use of an epoxy resin. A catalyst was applied on the electrode surface to establish equilibrium even further.

The sensor results are shown in Fig. 6. All Sensors exhibit the desired lambda switch. The one-step sintered sensor shows a lower signal compared to the joined sensor in the rich region, which cannot be explained by the temperature change alone. Also, an offset in the lean area can be seen for the one-step sintered sensor. Future upcoming work will address these issues. The commercial type LSH sensor and the joined sensor agree quite well. The lower values for the sensor voltage in rich gases may stem from the more advanced catalyst coating on the commercial sensor.

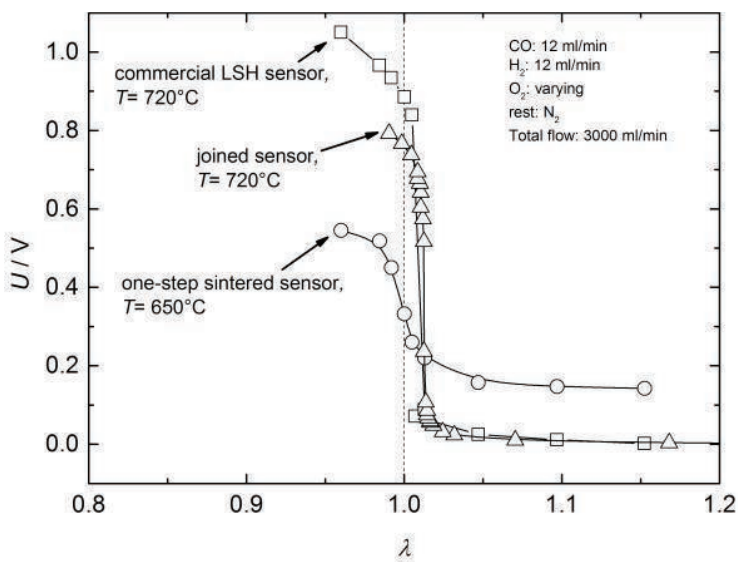

Fig. 6 Comparison between a commercial LSH type lambda probe, the joined sensor and a one-step sintered sensor to lambda variations around the stoichiometric point.

It is now possible to vary the material of both electrodes on a switching-type lambda sensor.
This may lead to many interesting prospects in future research and application.

\section{References}

[1] J. Riegel, Exhaust gas sensors for automotive emission control, Solid State Ionics 152-153, 783-800 (2002); doi: 10.1016/S01672738(02)00329-6

[2] T. Baunach, K. Schänzlin, L. Diehl, Sauberes Abgas durch Keramiksensoren, Physik Journal 5, 33-38 (2006)

[3] R. Moos, A Brief Overview on Automotive Exhaust Gas Sensors Based on Electroceramics, International Journal of Applied Ceramic Technology 2, 401-413 (2005); doi: 10.1111/j.1744-7402.2005.02041.x

[4] D. Schönauer, T. Nieder, K. Wiesner, M. Fleischer, R. Moos, Investigation of the electrode effects in mixed potential type ammonia exhaust gas sensors, Solid State lonics 192, 38-41 (2011); doi: 10.1016/j.ssi.2010.03.028 